\title{
A 1.5-approximation algorithm for augmenting edge-connectivity of a graph from 1 to $2^{*}$
}

\author{
Guy Even \\ Tel-Aviv University \\ guy@eng.tau.ac.il
}

\author{
Guy Kortsarz ${ }^{\dagger}$ \\ Rutgers University, Camden \\ guyk@crab.rutgers . edu
}

\author{
Zeev Nutov \\ The Open University of Israel \\ nutov@openu.ac.il
}

\begin{abstract}
We present a 1.5-approximation algorithm for the following NP-hard problem: given a connected graph $G=(V, \mathcal{E})$ and an edge set $E$ on $V$ disjoint to $\mathcal{E}$, find a minimum size subset of edges $F \subseteq E$ such that $(V, \mathcal{E} \cup F)$ is 2-edge-connected. Our result improves and significantly simplifies the approximation algorithm with ratio $1.875+\varepsilon$ of Nagamochi.
\end{abstract}

\section{Introduction}

\subsection{Problem definition and our result}

A graph (possibly with parallel edges) is $k$-edge-connected if there are $k$ pairwise edge-disjoint paths between every pair of its nodes. We study the following fundamental connectivity augmentation problem: given a connected undirected graph $G=(V, \mathcal{E})$ and a set of additional edges (called "links") $E$ on $V$ disjoint to $\mathcal{E}$, find a minimum size edge set $F \subseteq E$ so that $G+F=(V, \mathcal{E} \cup F)$ is 2-edge-connected. The 2-edge-connected components of the given graph $G$ form a tree. It follows that by contracting these components, one may assume that $G$ is a tree. Hence, our problem is:

Tree Augmentation Problem (TAP)

Instance: A tree $T=(V, \mathcal{E})$ and a set of links $E$ on $V$ disjoint to $\mathcal{E}$.

Objective: Find a minimum size subset $F \subseteq E$ of edges such that $T \cup F$ is 2-edge-connected.

TAP is sometimes posed as the problem of covering a laminar family (see e.g. [1]). Namely, given a laminar family $\mathcal{E}$ on a groundset $V$, and an edge set $E$ on $V$, find a minimum size $F \subseteq E$ such that for every $S \in \mathcal{E}$, there is an edge in $F$ with one endpoint in $S$ and the other in $V-S$. TAP is also equivalent to the problem of augmenting the edge-connectivity from $k$ to $k+1$ for any odd $k$; this is since the family of minimal cuts of a $k$-connected graph with $k$ odd is laminar.

\footnotetext{
*Preliminary version in APPROX 2001, pp. 90-101.

${ }^{\dagger}$ Partially supported by NSF grant number 0728787 .
} 
The first 2-approximation for TAP (which also holds for the weighted version) was given by Frederickson \& Jájá [4]. TAP is APX-hard even if the set $E$ of links forms a cycle on the leaves of $T$ [1]. Improving the approximation ratio below 2 was a long-standing open problem posed by Khuller in [8] as one of the main open problems in connectivity augmentation. The first approximation ratio below 2 was given by Nagamochi [11]; he gave a $(1.875+\varepsilon)$-approximation scheme for TAP. In the conference version [3] we presented and sketched a proof of a 1.5-approximation algorithm for the problem. Here we give a full and substantially simplified proof of this result.

Theorem 1.1 TAP admits a 1.5-approximation algorithm.

Several ideas introduced by Nagamochi in [11] are used in this paper (e.g., minimally leaf-closed trees and some parts of the lower bound). Key to our analysis is a credit scheme for using the different parts of the lower bound. This credit scheme has a "static" component and a "dynamic" component. The static credit is computed at the beginning of the algorithm and is distributed to different parts of the graph. The dynamic credit is available only after the algorithm "reveals" it by proving that the optimum solution had to be larger than the initial static estimate. We believe that this technique has merit of its own and may be useful for other connectivity problems.

\subsection{Related work}

Several particular cases of TAP were considered in the literature. Eswaran \& Tarjan [2] presented a linear time algorithm for the case when any link is legal, namely when $E$ forms a complete graph. A more general problem than TAP is the minimum weight 2-Edge-Connected Spanning Subgraph (2ECSS) problem, where the goal is to find a minimum weight 2-edge-connected spanning subgraph $H$ of a given complete graph $G$. TAP is a particular case, when $G$ contains a tree $T$ (or a connected graph) of weight 0 , and any other edge in $G$ has weight 1 .

A problem sandwiched between 2-ECSS and TAP is weighted TAP, when the edges in $E$ have weights and the goal is to find a minimum weight augmenting edge set. There are few 2approximation algorithms for weighted TAP. The first algorithm, by Frederickson and Jájá [4] was simplified later by Khuller and Thurimella [9]. These algorithms are based on constructing a directed graph and computing a minimum weight arborescence. The primal-dual algorithm of [6] is another simple combinatorial 2-approximation algorithm for the problem. The iterative rounding algorithm of Jain [7] is an LP-based 2-approximation algorithms. The approximation ratio of 2 for all these algorithms is tight even for TAP. However, while TAP admits an approximation algorithm with ratio better than 2 , no such algorithm is known for weighted TAP.

The 2-ECSS problem was vastly studied. For general weights, the best known ratio is 2 by Fredrickson and Jájá [4], which can also be achieved by the the algorithms in [9] and [7]. For particular cases, better ratios are known. Fredrickson and Jájá [5] showed that when the edge weights satisfy the triangle inequality, the Christofides heuristic leads to a 3/2-approximation algorithm. For the special case of 2-ECSS in which edges have $\{1, \infty\}$ weights, the currently best known ratio 
is $4 / 3$ [12]. Surveys on broad classes of connectivity problems can be found in [8] and [10].

\section{Preliminaries}

Let $T=(V, \mathcal{E})$ be a tree. For $u, v \in V$ let $(u, v) \in \mathcal{E}$ denote the edge in $T$ and $u v$ the link in $E$ between $u$ and $v$. Let $p(u v)$ denote the path between $u$ and $v$ in $T$. A link $u v$ covers all the edges (and nodes) along the path $p(u v)$. We designate a node $r$ of $T$ as the root, and refer to the pair $T, r$ as a rooted tree (we do not mention the root when it is clear from the context). The choice of $r$ defines a partial order on $V: u$ is a descendant of $v$ (or $v$ is an ancestor of $u$ ) if $v$ belongs to $p(r u$ ); if, in addition, $(u, v) \in T$, then $u$ is a child of $v$, and $v$ is the parent of $u$. The leaves of $T$ are the nodes in $V-r$ that have no descendants. We denote the leaf set of $T$ by $L(T)$, or simply by $L$, when the context is clear. The rooted subtree of $T$ induced by $v$ and its descendants is denoted by $T_{v}\left(v\right.$ is the root of $T_{v}$ ). A subtree $T^{\prime}$ of $T$ is called a rooted subtree of $T$ if $T^{\prime}=T_{v}$ for some $v \in V$.

To contract a rooted subtree $T^{\prime}$ of $T$ is to combine all nodes in $T^{\prime}$ into a single node $v$. The edges and links with both endpoints in $T^{\prime}$ are deleted. The edges and links with one endpoint in $T^{\prime}$ now have $v$ as their new endpoint

If we add a link $u v$ to a partial solution $I$, then the nodes along the path $p(u v)$ belong to the same 2-edge-connected component of the augmented graph $(V, \mathcal{E} \cup I)$. Hence, we may contract the nodes along $p(u v)$. For a set of links $I \subseteq E$, let $T / I$ denote the tree obtained by contracting every 2-edge-connected component of $T \cup I$ into a single node. Since all contractions are induced by subsets of links, we refer to the contraction of every 2-edge-connected component of $T \cup I$ into a single node simply as the contraction of the links in $I$.

Definition 2.1 A link $u^{\prime} v^{\prime}$ is a shadow of a link uv if $p\left(u^{\prime} v^{\prime}\right) \subseteq p(u v)$. A link is maximal if it is not a shadow of any other link. An edge $e$ of $T$ is reducible with respect to a link set $E$ if one of the following holds:

(i) There is a unique maximal link that covers e.

(ii) There exists an edge $e^{\prime} \neq e$ such that every maximal link that covers $e^{\prime}$ also covers $e$.

If an edge $(u, v)$ is covered by a unique maximal link $a b$, then taking $a b$ to the cover is an optimal step (i.e., the size of an optimal solution in the residual problem is smaller by one). If an edge $e$ is covered by any maximal link that covers another edge $e^{\prime} \neq e$, then $e$ may be contracted without affecting the sizes of the optimal solution and the computed solution (as we still need to cover $e^{\prime}$ ). Hence, we may pre-process the instance so that:

Assumption 2.1 There are no reducible edges in the tree $T$ with respect to the link set $E$.

The following statement shows that if there are no reducible edges, then paths in $T$ consisting of degree 2 nodes can not end at leaves.

Claim 2.2 If there are no reducible edges, then every parent of a leaf has at least two children. 
Proof: For the sake of contradiction, suppose that $v$ has a unique leaf child $a$ in $T$. Let $u$ the parent of $v$. Thus $v$ has degree exactly 2. Note that if there is a link $a v \in E$ parallel to the edge $(a, v)$, then $a v$ can not be the only link incident to $a$, as otherwise the edge $(a, v)$ is reducible. Hence $a v$ is not maximal. Thus any maximal link incident to $a$ covers the edge $(u, v)$, which contradicts the assumption that $T$ has no reducible edges.

Definition 2.2 $A$ node $s \in V-r$ is a stem of $T$ if it has exactly two children and both of them are leaves. The link between the two children of a stem, if it exists, is called a twin-link.

Claim 2.3 If no edge of $T$ is reducible then there is a twin-link between the children of every stem.

Proof: Let $s$ be a stem with children $a, b$, and let $v$ be the parent of $s$. If $a b \notin E$, then since there are at least two links covering the edge $(a, s)$ (because no edge is reducible), every maximal link that covers $(a, s)$ also covers the edge $(s, v)$. Hence, $(s, v)$ is reducible, contradicting the assumption.

Every TAP instance can be rendered closed under shadows by adding all shadows of existing links. We refer to the addition of all shadows as shadow completion. Shadow completion does not affect the optimal solution size, since every shadow can be replaced by some link covering all edges covered by the shadow. Thus:

Assumption 2.4 The set of links $E$ is closed under shadows, that is, if $u v \in E$ and $p\left(u^{\prime} v^{\prime}\right) \subseteq p(u v)$ then $u^{\prime} v^{\prime} \in E$.

Definition 2.3 A cover $F$ of $T$ is shadows minimal if for every link uv $\in F$ replacing uv by a proper shadow of uv results in a set of links that does not cover $T$. The tree $T^{\prime}$ is proper if there exists a minimum cover of $T^{\prime}$, that contains no (parallel) link between a stem and its child.

Claim 2.5 Let $F$ be a shadows minimal cover of $T$. Then there is exactly one link in $F$ incident to every leaf of $T$. In particular, the leaf-to-leaf links in $F$ form a matching on $L(T)$.

Proof: Let $a$ be a leaf. If $a u, a v \in F$ for $u \neq v$, then we can replace the link $a v$ by its shadow $a^{\prime} v$ where $a^{\prime}$ is the parent of $a$. Since every edge of $T$ remains covered, this contradicts the shadows minimality of $F$. The statement follows.

Claim 2.6 Under Assumptions 2.1 and 2.4 there exists an optimal cover of $T$ that is proper.

Proof: We only need to show that there is a shadows minimal optimum solution $F$ that contains no link from a twin to its stem brother. Let $F$ be an optimal shadows minimal cover of $T$ with maximal number of twin links. Let $s$ be a stem with children $a, b$ and suppose to the contrary that $a s \in F$. Let $b v$ be the (unique, by Claim 2.5) link in $F$ that is incident to $b$. Note that $a b \in E$ (by Claim 2.3), and that if $v \neq s$ then $s v$ is a link (by Assumption 2.4). Let

Consider $F^{\prime}=(F \backslash\{a s, b v\}) \cup\{a b\}$ if $v=s$ and $F^{\prime}=(F \backslash\{a s, b v\}) \cup\{a b, s v\}$ if $v \neq s$. Then $F^{\prime}$ is a shadow minimal cover of $T,\left|F^{\prime}\right| \leq|F|$, but $F^{\prime}$ has more twin links than $F$. This contradicts our choice of $F$. 
For $X, Y \subseteq V$ and a link set $F$, let $F(X, Y)=\{x y \in F: x \in X, y \in Y\}$ denote the set of links in $F$ that have one endpoint in $X$ and the other in $Y$; for $x \in V$ let $\operatorname{deg}_{F}(x)=|F(x, V)|$ be the degree of $x$ w.r.t. $F$. For the rest of the paper, assume that Assumptions 2.1 and 2.4 are valid, and fix $F$ to be some optimal proper solution for an instance $T=(V, \mathcal{E}), E$ of TAP.

\section{The lower bound and the algorithm}

\subsection{The Lower Bound}

Lemma 3.1 Let $L$ be the set of leaves and $S$ be the set of stems of $T$, let $X=V \backslash(L \cup S)$, and let $F$ be a proper cover of $T$. Then

$$
|F| \geq \frac{2}{3}|L|-\frac{1}{3}\left|M_{F}\right|+\frac{1}{3} \sum_{x \in X \cap T^{\prime}} \operatorname{deg}_{F}(x) .
$$

Proof: Let $\hat{M}_{F}$ be the set of twin links in $F$. Note that $M_{F} \cup \hat{M}_{F}$ is a matching, by Claim 2.5. Partition $F$ into $M_{F} \cup \hat{M}_{F}$ and all possible combinations of endpoints in $L, S$ and $X$ to get:

$$
|F|=\left|M_{F}\right|+\left|\hat{M}_{F}\right|+|F(L, S)|+|F(L, X)|+|F(S, S)|+|F(S, X)|+|F(X, X)| .
$$

Clearly

$$
|L|=2\left(\left|M_{F}\right|+\left|\hat{M}_{F}\right|\right)+|F(L, S)|+|F(L, X)| .
$$

Note that if $a, b$ are children of a stem $s$ and $a b \in \hat{M}_{F}$, then $\operatorname{deg}_{F}(s) \geq 1$. Therefore,

$$
\left|\hat{M}_{F}\right| \leq|F(L, S)|+2|F(S, S)|+|F(S, X)| .
$$

Multiply (2) by $2 / 3$, multiply (3) by $1 / 3$, and then add and rearrange the terms to get:

$$
\begin{aligned}
\frac{2}{3}|L|-\frac{1}{3}\left|M_{F}\right| & \leq\left|M_{F}\right|+\left|\hat{M}_{F}\right|+F(L, S)+\frac{2}{3}|F(L, X)|+\frac{2}{3}|F(S, S)|+\frac{1}{3}|F(S, X)| \leq \\
& \leq|F|-\frac{1}{3}(|F(L, X)|+|F(S, X)|+2|F(X, X)|)=|F|-\frac{1}{3} \sum_{x \in X \cap T^{\prime}} \operatorname{deg}_{F}(x) .
\end{aligned}
$$

The following corollary is implied from Corollary 3.3.

\section{Corollary 3.2}

$$
|F| \geq \frac{2}{3}|L|-\frac{1}{3}\left|M_{F}\right|+\mu / 3+\frac{1}{3} \sum_{x \in X \cap T^{\prime}} \operatorname{deg}_{F}(x) .
$$

If $M$ takes many links that have two leaf and one twin, this may complicate the analysis, and so we define the following matching $M$ (that is easily seen to be computable in polynomial time).

Definition 3.1 A mixed link is a link from a twin leaf to a non twin leaf. Let $M$ be such that 
1. $M$ is a maximum matching among matchings that do not contain twin links.

2. Among matchings as above, $M$ contains the least mixed edges. And among all such matching $M$ contains the least mixed leaves.

Definition 3.2 We say that a pairs of leaves $x, y$ are $(F, M)$-bad if $x y \in M$. and $x, y$ are either not matched in $F$ or both belong to twin links $F$ took.

Say that there exist $\mu$ pairwise node disjoint $(F, M)$-bad pairs. Let $M_{F}$ be the set of leaf-to-leaf links in $F$ that are not twin links.

Claim 3.3 $M \geq M_{F}+\mu$.

Proof: The bad pairs are node disjoint. Let $x, y$ be a bad pair. Note that for every bad pair increases $M$ by 1 but does not increase $M_{F}$ by 1 because $M_{F}$ contains no twin links. Thus every bad pair implies that $M$ is larger than $M_{F}$ by one more unit. The claim follows.

\subsection{The credit scheme and the invariants of the algorithm}

We explain how we use the lower bound. Let $U$ denote the set of leaves unmatched by $M$, so $|U|=|L|-2|M|$. By multiplying both sides of the inequality in Corollary 3.2 by 1.5 and rearranging terms, we obtain

$$
\frac{3}{2} \cdot|F| \geq|U|+\frac{3}{2} \cdot|M|+\mu / 2+\frac{1}{2} \sum_{x \in X \cap T^{\prime}} \operatorname{deg}_{F}(x)
$$

We prove the following statement that implies Theorem 1.1.

Theorem 3.4 There exists a polynomial time algorithm that for an instance of TAP computes a solution I of size at most the right-hand size of (5). Thus $|I| \leq 1.5 \cdot|F|$.

Initially, the algorithm assigns coupons to unmatched leaves and to links in $M$ so that the total number of coupons assigned equals the sum of the first two terms in the right hand side of (5). For technical reasons, we also assign 1 coupon to the root $r$.

Definition 3.3 A node that contains several nodes is called a compound node.

\section{Initial assignment of coupons:}

- Every unmatched leaf gets 1 coupon, and $r$ gets 1 coupon.

- Every link in $M$ gets $3 / 2$ coupons.

\section{Invariant 3.5 (Coupons Invariant)}

1. Every unmatched leaf of $T / I$ owns a coupon.

2. Every compound node owns a coupon. 
3. Every link in $M$ owns $3 / 2$ coupons. Also $r$ owns one coupon.

Let $C\left(T^{\prime}\right)$ be the non leaves compound nodes. The root $r$ is also considered a compound node.

Tickets. The following assignment of tickets depends on $F$ and is not known to the algorithm.

1. An $(F, M)$-bad pair $b_{1} b_{2}$ is assigned a matching ticket, that is worth $1 / 2$ a coupon, and this ticket is located at $\left\{b_{1} b_{2}\right\}$.

2. A node $x \in X \cap T^{\prime}$ that is linked in $F$ is assigned a ticket (1/2 coupon) at $x$.

To charge the last term $\mu / 2+\frac{1}{2} \sum_{X} \operatorname{deg}_{F}(x)$ we must show, for example, that a node in $X$ is touched by a link in $F$ or that there is an $F, M$ bad pair $b_{1} b_{2}$.

Definition 3.4 A matching is proper, if the contraction of any set of links in $M$ does not create a leaf.

\section{Invariant 3.6 (Matching Invariant)}

1. $M$ is a matching on non-compound leaves of $T / I$ without twin links and without locking links.

2. A pair of bad leaves for $F, M$ contains a matching ticket that is worth $1 / 2$ a coupon.

3. $M$ is a proper matching

\section{Rule 1: Claiming every ticket at most once}

(a) Tickets are claimed only in a rooted subtree $T^{\prime}$ of $T / I$.

(b) Tickets are never claimed on compound nodes.

(c) After a ticket is claimed at $\mathrm{x}, x$ enters into a compound node.

Properties $b, c$ assure that a ticket is claim at a node at most one time.

Summarizing, we use the following notation for the credit distributed in a rooted subtree $T^{\prime}$ of $T / I$. Let coupons $\left(T^{\prime}\right)$ denote the total number of coupons owned by $T^{\prime}$; this includes the coupons owned by nodes of $T^{\prime}$ (every unmatched leaf owns a coupon), and 3/2 coupons for every link in $M$ with both endnodes in $T^{\prime}$. Let tickets $\left(T^{\prime}\right)=\mu / 2+\sum_{x \in X \cap T^{\prime}} \operatorname{deg}_{F}(x)$ denote the number of tickets in $T^{\prime}$. Let $\operatorname{credit}\left(T^{\prime}\right)=\operatorname{coupons}\left(T^{\prime}\right)+\operatorname{tickets}\left(T^{\prime}\right)$.

\subsection{The main ideas of the algorithm}

Our goal is to iteratively find a tree $B^{\prime}$ and a cover $B^{\prime}$ so that $\left|B^{\prime}\right|=\operatorname{credit}\left(T^{\prime}\right)-1$. In this case, we can leave a coupon in the newly created leaf, in order to Maintain Item 2 in the coupons invariant. 
Except for one case, in which the tree is called deficient we can directly find such a tree. In case of a deficient tree we still find a tree as above, but it would require some additional computations.

We shall later see that if $T^{\prime}$ has a ticket, we can find a cover $B^{\prime}$ of $T^{\prime}$ so that $\operatorname{credit}\left(T^{\prime}\right) \geq B^{\prime}+1$ holds. However, the question is how can we show that the tree has a ticket when we do not know $F$ ? Our idea is to perform an exhaustive search over all the possible sets $F \cap T^{\prime}$ that cover $T^{\prime}$, and show that $T^{\prime}$ contains a ticket, regardless of the chosen $F \cap T^{\prime}$ Thus we can cover $T^{\prime}$ by the basic cover and leave a coupon. Note that we do not need to know where is the ticket to do that, but only need the proof that for every possible choice of $F \cap T^{\prime}, T^{\prime}$ contains a ticket.

Definition 3.5 ([11]) Let $U$ be a subset of nodes of $T$. A rooted subtree $T^{\prime}$ of $T$ is $U$-closed if there is no link in $E$ from $U \cap T^{\prime}$ to $T \backslash T^{\prime} . T^{\prime}$ is leaf-closed if it is $L(T)$-closed. A leaf-closed $T^{\prime}$ is minimally leaf-closed if any proper rooted subtree of $T^{\prime}$ is not leaf-closed. For a single vertex $x$ we just say that $T^{\prime}$ is $x$-closed.

For a node set $U \subseteq V$, we let $u p(U)=\{u p(u): u \in U\}$.

Proposition 3.7 ([11]) A minimally leaf-closed subtree $T^{\prime}$ of $T$ is covered by up $\left(L\left(T^{\prime}\right)\right)$.

The problematic (namely deficient) trees are defined as follows.

Definition 3.6 Deficient tree $A$ tree is deficient if it contains 3 leaves, $b_{1}, b_{2}, a_{1}$ so that $b_{1} b_{2}$ is a link, and $T^{\prime}$ is $a_{1}$-closed, and in addition we can order $b_{1} b_{2}$ in one of the two orders, say $b_{1} b_{2}$ so that:

1. $a_{1} b_{1}$ is a link and its contraction does not create a new leaf.

2. The tree is not $b_{2}$-closed.

\subsection{The algorithm}

Our approach is to iteratively find non deficient tree, contract it and leave a coupon (in order to satisfy the Coupons Invariant 3.5). Thus, at the end $T$ is completely covered, The number of links we use is at most the right hand side of Inequality (5), as claimed in Theorem 3.4.

The following lemma is the main one to prove, and it is proved using various claims.

Lemma 3.8 Suppose that Invariants 3.5 and 3.6 hold for $T, M$, and $I$. Then there exists a polynomial time algorithm that finds non deficient subtree $T^{\prime}$ of $T / I$.

Algorithm Tree-Cover $(T=(V, \mathcal{E}), E)$ (A 1.5-approximation algorithm)

1. initialize: $I \leftarrow \emptyset$

2. Eliminate reducible edges of $T$ and update $I$ accordingly.

3. Let $M$ be a matching as in Definition 3.1. 
4. Give 1 coupon to every unmatched leaf and to $r$, and give $3 / 2$ coupons to every link in $M$.

5. While $T / I$ has more than one node do:

(a) Greedy steps: As long as there is a link that covers a path with two coupons add this link to $I$

(b) Find a non deficient subtree $T^{\prime}$ of $T / I$ with cover $B^{\prime}$ of $T^{\prime}$ as in Lemma 3.8. Contract $T^{\prime}$, assign 1 coupon to the new compound leaf, and set $I \leftarrow I \cup B^{\prime}$.

6. Return $I$

Algorithm Tree-Cover initiates $I \leftarrow \emptyset$ as a partial cover. It applies the reductions from Section 2 (specifically, we need Assumption 2.1) computes a maximum matching $M$ as in Definition 3.1 and initiates the credit scheme as described in Section 3.2. In the main loop, the algorithm iteratively exhausts greedy contractions, then it computes $T^{\prime}, B^{\prime}$ as in Lemma 3.8, adds $B^{\prime}$ to $I$, contracts $T^{\prime}$, and leaves a coupon on the created compound leaf. The stopping condition is when $I$ covers $T$, namely, when $T / I$ is a single node.

It is easy to verify that all actions needed to be taken by the algorithm can be implemented in polynomial time. The credit scheme used implies that the algorithm computes a solution $I$ as in Theorem 3.4, namely $|I|$ is at most 1.5 times the right-hand size of (5). Hence the approximation ratio of 1.5 follows from the lower bound and the proof of Theorem 1.1 is now complete.

\section{Proof of Lemma 3.8}

\subsection{Semi-closed trees and their properties}

Recall that $U p\left(T^{\prime}\right)$ covers all the leaves of a minimally closed tree $T^{\prime}$. A naive approach to find $T^{\prime}, B^{\prime}$ as in Lemma 3.8 is by taking $T^{\prime}$ to be minimally leaf-closed and $B^{\prime}=u p\left(L\left(T^{\prime}\right)\right)$. However, we do not have enough credit for that, since $\left|L\left(T^{\prime}\right)\right|$ can be much larger than coupons $\left(T^{\prime}\right)$, not to mention the one extra unit of credit. This is since every link $b_{1} b_{2} \in M$ owns $3 / 2$ coupons, while taking the up-links of $b_{1}, b_{2}$ requires 2 coupons. Hence we define $T^{\prime}$ and its cover $B^{\prime}$ so that $\operatorname{coupons}\left(T^{\prime}\right) \geq\left|B^{\prime}\right|$ holds, and one extra unit of credit is provided with the help of tickets. Our main new idea is to use minimally semi-closed trees, defined below, which admit such a cover $B^{\prime}$, assuming invariants from Section 3.2 hold.

Definition 4.1 (Minimally Semi-closed Tree) A rooted subtree $T^{\prime}$ of $T / I$ is minimally-semiclosed (w.r.t. a proper matching $M$ ) if the following holds:

- For any $b_{1} b_{2} \in M$ either both $b_{1}, b_{2}$ belong to $T^{\prime}$, or none of $b_{1}, b_{2}$ belongs to $T^{\prime}$.

- $T^{\prime}$ is closed w.r.t. its unmatched leaves.

- There is no proper subtree of $T^{\prime}$ with the above properties. 
Note that a minimally-semi-closed $T^{\prime}$ is not leaf-closed (this why we called it "semi-closed"); $T^{\prime}$ is closed with respect to every unmatched leaf, but matched leaves may have links to nodes outside $T^{\prime}$. In what follows, let us use the following notation:

- $M\left(T^{\prime}\right)$ is the set of links in $M$ with both endnodes in $T^{\prime}$.

- $U\left(T^{\prime}\right)$ is the set of unmatched leaves of $T^{\prime}$.

- $C\left(T^{\prime}\right)$ is the set of non-leaf compound nodes of $T^{\prime}$ (recall that this includes $r$, if $r \in T^{\prime}$ ).

- $B\left(T^{\prime}\right)=M\left(T^{\prime}\right) \bigcup u p\left(U\left(T^{\prime}\right)\right)$.

Lemma 4.1 If all greedy contractions are exhausted, then $M$ is a proper matching. This keep the proper matching invariant.

Proof: Let $a b \in M$. Note that in the original tree $T$ the contraction of $a b$ does not create a leaf, since $M$ contains no twin links. Thus, if the contraction of $a b$ creates a leaf in $T / I$, then $p(a b)$ contains a compound node in $T / I$. This holds as some structure that hanged out of a node of $p(a b)$ and contained a leaf is now gone (this structure was avoiding the contraction of $a b$ in $T$ from creating a new leaf). But then $a b$ gives a greedy contraction, contradicting the assumption.

The following statement explains how we intend to cover minimally semi-closed trees.

Lemma 4.2 If $T^{\prime}$ is minimally semi-closed then $B\left(T^{\prime}\right)$ covers $T^{\prime}$.

Proof: Let $T^{\prime \prime}$ be obtained by contracting $M\left(T^{\prime}\right)$. The contraction of $M\left(T^{\prime}\right)$ does not create a new leaf, as the matching is proper. Thus $L\left(T^{\prime \prime}\right)=U\left(T^{\prime}\right)$, Therefore, $T^{\prime \prime}$ is minimally leaf-closed, since $T^{\prime}$ is minimally semi-closed. Thus $u p\left(U\left(T^{\prime}\right)\right.$ ) covers $T^{\prime \prime}$ (if $T^{\prime}$ has no unmatched leaves then $T^{\prime \prime}$ is a single node), by Proposition 3.7. The statement follows.

Definition 4.2 $B\left(T^{\prime}\right)$ is called the basic cover of $T^{\prime}$

\subsection{Some preliminary claims}

Claim 4.3 If $\mid M\left(T^{\prime}\right) \geq 2, T^{\prime}$ is non deficient. Also, if the tree contains a ticket, it is non deficient. Finally, if $C\left(T^{\prime}\right) \neq \emptyset$, the tree is not deficient.

Proof: Every contracted matched pair leaves 0.5 unused coupons because it has 1.5 coupons and one coupon only is required to contract it. Thus if $\left|M\left(T^{\prime}\right)\right| \geq 2$, the tree is not deficient. If $\left|M\left(T^{\prime}\right)\right|=1$ and $T^{\prime}$ contains a ticket, the 0.5 left from the unique matched link and the $1 / 2$ a coupon from the ticket, give 1 coupon that can be left at the new leaf, hence the tree is non deficient. Also, if $C\left(T^{\prime}\right) \neq \emptyset$, clearly the tree is not deficient.

Claim 4.4 If the tree $T^{\prime}$ has 3 leaves, a matched pair $b_{1} b_{2}$ and a third leaf $a_{1}$ but the tree is non deficient, we can cover the tree $T^{\prime}$ and leave a coupon

Proof: First say that the tree has no stem The unmatched leaf $a_{1}$ must be covered with a link to $b_{1}$ or $b_{2}\left(\right.$ as $\left.C\left(T^{\prime}\right)=\emptyset\right)$. But if $a_{1}$ is covered in $F$ by, say, $a_{1} b_{1}$, as the tree is non deficient, $b_{2}$ is 
$T^{\prime}$-closed and we get a ticket in order to cover the root.

Recall that a mixed edge contains a twin and a non twin leaves. In case there is a stem, the mixed edge $b_{1} b_{2}$ is in $M$. In case $F$ took the twin link, we show that $b_{1} b_{2}$ is an $(F, M)$-bad pair, and thus the tree contains a matching ticket. Note that as the tree has 3 leaves, and two of them are already matched $b_{2}$ can not be linked to a leaf in $T^{\prime}$.

Clearly, $b_{2}$ can be linked outside $T^{\prime}$. But the other endpoint of the link that touches $b_{2}$ is not a leaf, because $T^{\prime}$ does not split leaves. Thus, $b_{2}$ is unmatched, and $b_{1}$ is covered by a twin link and thus, by definition, we get a matching ticket.

Now say that $F$ did not take the twin link. The only way to avoid a ticket, is to link $a_{1}$ to $b_{2}$. By Claim 4.7 this means that $F$ has no links touching the stem $s$. Thus to avoid a ticket for covering the root, it must be that $T^{\prime}$ is not $b_{1}$-closed. But this implies that the tree is deficient, contradiction.

By Claim 4.4 we get

Corollary 4.5 If the tree is non deficient, we may assume that $T^{\prime}$ has at least 4 leaves

Thus we shall assume the tree has at least 4 leaves, until we separately deal with deficient trees.

\subsection{Help nodes}

Note that unmatched leaves in $M$ in a minimally semi-closed tree $T^{\prime}$ are $T^{\prime}$-closed. Thus for an unmatched leaf $a$ the other endpoint of the tree covering it belongs to $T^{\prime}$.

Definition 4.3 A help node in $F$ is a node $x$ that is either a stem, or a matched leaf in $M$ that is connected to some unmatched in $M$ leaf $a$. In such a case we say that $x$ helps a (namely, helps a to avoid a ticket).

Note that if an unmatched leaf is not linked to a help node, $T^{\prime}$ has a ticket and we are done.

Notation 4.6 We denote the number of matched nodes, plus the number of stems by $h$.

Claim 4.7 If $T^{\prime}$ contains a stem and $F$ contains a mixed link then $s$ can not help the $\left|U\left(T^{\prime}\right)\right|$ unmatched nodes. Also if a pair $x y$ is matched in $F, x$ and $y$ can not help other uncovered leaves.

Proof: The first claim follows by shadow minimality. The second claim follows because leaves have degree 1 in $F$.

Claim 4.8 $T^{\prime}$ has at least $\left|U\left(T^{\prime}\right)\right|-h+1$ tickets.

Proof: Consider the node that covers the root. If a stem covers the root, by shadow minimality it can not be connected to any of the $\left|U\left(T^{\prime}\right)\right|$ unmatched leaves and we loose a help node. If a node matched by $M$ covers the root in $F$, as its degree in $F$ is 1 , this node can not be a help node. Thus in any case, we loose a help node, for covering the root, and the number of help nodes is therefore 
at most $h-1$. The claim follows.

Claim 4.9 If the tree has no matched pair then it has two tickets.

Proof: Note that the tree can not contain a stem by Claim 4.11. As all leaves are unmatched, it means that all leaves are independent with respect to links because no greedy operation is possible. Thus, $h=0$ (no matched pairs, no stems so no help nodes). As $\left|U\left(T^{\prime}\right)\right| \geq 1$, the proof follows from Claim 4.8 .

By Claims 4.3 and 4.9 we get:

Assumption $4.10\left|M\left(T^{\prime}\right)\right|=1$

Claim 4.11 A stem not destroyed by greedy, it has has one matched leaf and one unmatched leaf Proof: If non of the two twins are unmatched, the twin link is added via a 1-greedy operation and the stem is gone. If both leaves are matched, $\left|M\left(T^{\prime}\right)\right| \geq 2$. The claim follows.

\subsection{The case of two stems or more}

Claim 4.12 If the tree contains two stems or more the tree is non deficient

Proof: If the tree contains 3 stems or more it contains two matched links in $M$ and it is a non deficient tree. Thus we may assume that the tree contains exactly two stems. The tree has one matched link. The links has to be $b_{1} b_{2}$ so that $b_{1}$ is a child of $s_{1}$ and $b_{2}$ is a child of $s_{2}$ (in any other case $\left|M\left(T^{\prime}\right)\right|=1$.

If $F$ takes the both twin links, we get a matching ticket, and we are done. Indeed, both $b_{1}, b_{2}$ are matched in $M$ but not in $M_{F}$. Note that the matching tickets we discover are disjoint because we will contract $T^{\prime}$ by its basic cover.

Now consider the case that $F$ takes one twin link $a_{1} b_{1}$ but it does not take $a_{2} b_{2}$. Note that $a_{2} s_{1}$ can not be a link as otherwise, its shadow $a_{1} a_{2}$ exists and we get a greedy step. Also as leaves have degree 1 with respect to $F, a_{2}$ can not be connected to $b_{1}$. And the link $a_{1} a_{2}$ does not exist. This gives a ticket for covering $a_{2}$. The last case is that $F$ does not take any twin links. The only way to avoid a tickets is if $a_{1} b_{2}$ and $a_{2} b_{1}$ exist, and belong to $F$. By Claim 4.7, in this case the stems have no links in $F$. Thus $h=2$, namely the two nodes $b_{1}, b_{2}$ and $\left|U\left(T^{\prime}\right)\right|=2$. The claim follows from Claim 4.8.

Claim 4.13 If the tree contains 5 leaves it has a ticket

Proof: In this case $\left|U\left(T^{\prime}\right)\right|=3$ because only two leaves are matched in $M$. Also $h \leq 3: b_{1}, b_{2}$ and a possible unique stem can be help nodes. The Claim follows from Lemma 4.8. 


\subsection{Exactly four leaves and exactly and one stem}

The four leaves are denoted by $b_{1}, b_{2}, a_{1}, a_{2}$ with $a_{1} b_{1}$ being the twins.

Claim $4.14 a_{2} b_{2}$ can not be a link

Proof: If $a_{2} b_{2}$ is a link, replace $b_{1} b_{2} \in M$ by $a_{2} b_{2}$. The size of the matching did not change. But we have one less mixed link. This contradicts the definition of $M$

Claim 4.15 If a (non deficient) the tree contains a stem, it has a ticket.

Proof: If $F$ contains mixed edge, then the stem is not linked in $F$ by Claim 4.7. Thus $h \leq 2$, because only the matched pair $b_{1}, b_{2}$ can be help nodes. Clearly, $\left|U\left(T^{\prime}\right)\right|=2$. In such a case the claim follows from Claim 4.8. Now say that $F$ does not contain a mixed edge. We claim that in such case $F$ has no links at all in $T^{\prime}$. Indeed, the only possible link in $F$ link of another type is $a_{2} b_{2}$. But such a link does not exist. As $F$ has no links in $T^{\prime}$, one of that ways to get a ticket is to observe that $b_{1} b_{2}$ gives a matching ticket.

Claim 4.16 If $T^{\prime}$ contains no stems, it has a ticket

Proof: Note that $\left|U\left(T^{\prime}\right)\right|=2$ and $h=2\left(b_{1}, b_{2}\right.$ can be help nodes). The claim follows from Claim 4.8

As we went over all cases, This ends the proof of Lemma 3.8. The proof is complete.

\subsection{Dealing with Deficient trees}

Acknowledgment: We thank André Linhares, Zhihan Gao, Joseph Cheriyan, Chaitanya Swamy, and two anonymous referees for many useful comments, and Jon Feldman for some useful discussions. We also thank Samir Khuller for simplifying the proof of Corollary 3.2.

\section{References}

[1] J. Cheriyan, T. Jordán, and R. Ravi. On 2-coverings and 2-packing of laminar families. In ESA, pages 510-520, 1999.

[2] K. Eswaran and R. Tarjan. Augmentation problems. SIAM J. Computing, 5:653-665, 1976.

[3] G. Even, J. Feldman, G. Kortsarz, and Z. Nutov. A 3/2-approximation for augmenting a connected graph into a two-connected graph. In APPROX, pages 90-101, 2001.

[4] G. N. Frederickson and J. Jájá. Approximation algorithms for several graph augmentation problems. SIAM J. Computing, 10:270-283, 1981. 
[5] G. N. Frederickson and J. Jájá. On the relationship between the biconnectivity augmentation and traveling salesman problem. Theoretical Computer Science, 19(2):189-201, 1982.

[6] M. Goemans, A. Goldberg, S. Plotkin, E. T. D. Shmoys, and D. Williamson. Improved approximation algorithms for network design problems. In SODA, pages 223-232, 1994.

[7] K. Jain. A factor 2 approximation algorithm for the generalized steiner network problem. Combinatorica, 21(1):39-60, 2001.

[8] S. Khuller. Approximation algorithms for finding highly connected subgraphs (chapter 6). In Approximation algorithms for NP-hard problems (Ed. D. S. Hochbaum). PWS, Boston, 1996.

[9] S. Khuller and R. Thurimella. Approximation algorithms for graph augmentation. J. of Algorithms, 14:214-225, 1993.

[10] G. Kortsarz and Z. Nutov. Approximating minimum cost connectivity problems (chapter 58). In Handbook of Approximation Algorithms and Metahueristics (Ed. T. F. Gonzales). Chapman \& Hall/CRC, 2007.

[11] H. Nagamochi. An approximation for finding a smallest 2-edge connected subgraph containing a specified spanning tree. Discrete Applied Mathematics, 126:83-113, 2003.

[12] A. Sebö and J. Vygen. Shorter tours by nicer ears: 7/5-approximation for graphic tsp, 3/2 for the path version, and 4/3 for two-edge-connected subgraphs. CoRR, abs/1201.1870v2, 2012. 